\title{
Effective SEM Analytical Techniques for the Cathodoluminescence Visualization of Intergranular Cements in Saint Peter Sandstone: A Round Robin Exercise
}

\author{
William Schneider ${ }^{1}$, Colin McRae $^{2}$, and John Fournelle ${ }^{1}$ \\ 1. Department of Geoscience, University of Wisconsin, Madison, WI 53706, USA \\ 2. CSIRO Mineral Resources, Microbeam Laboratory, Private Bag 10, Clayton South, Australia, 3169
}

The use of Cathodoluminescence (CL) as an analytical tool for geoscience and materials research is well established. Many minerals exhibit CL when activated by the high-energy electron beam bombardment of the Scanning Electron Microscope (SEM) [1]. CL imaging capabilities vary among laboratories due to the differences in detector type, sample preparation (epoxy, polish, coating), SEM type (W, LaB6, $\mathrm{FE})$ and analytical conditions $(\mathrm{kV}, \mathrm{pA} / \mathrm{nA}$, sample temperature). An interlaboratory comparison was initiated to assess the optimal conditions for obtaining high resolution CL imagery [2]. Quartz (and its polymorphs) are often difficult to image by CL due to low CL response and thus it was chosen as a test material. Composed of fine to medium-grained silicon dioxide, St. Peter Sandstone is used extensively as a proppant in hydraulic formation fracture (fracking) [3]. Appropriate sample preparation is necessary for optimal CL imaging results, varying from lab to lab. In the case of the porous St. Peter Sandstone with incomplete and fragile intergranular cement structures, producing a polished sample surface without plucking grains and removing the cements is imperative. For this interlaboratory comparison, polished samples were prepared from a single St. Peter Sandstone sample that was cored perpendicular to the bedding. Samples were imbedded in Epoxy and polished to a sub micron finish. Six of these samples were shared with other SEM-EPMA laboratories with the goal of refining SEM-CL conventions for cement study and imagery. As of abstract deadline time, we have 3 examples of CL images to present, and expect to have others by the M\&M conference.

An investigator at NIST acquired CL images on an FEI Quanta 200 FEG with a Gatan MonoCL4 Elite with HSPMT, at 4 voltages $(30,20,10,3 \mathrm{kV})$ and currents ranging from $78-167 \mathrm{pA}$. SE and CL images were acquired at $10 \mathrm{kV}$ (Figures 1 and 2). Darkening of the image near the edges is due to low magnification beam scan exceeding the collection optics effective response range. CSIRO was provided unimproved St. Peter Sandstone core material for special sample preparation. The CL images were acquired on a JEOL 8500F FEG Electron Microprobe using $15 \mathrm{kV}, 30 \mathrm{~ms}$ dwell, 1 micron step with 1 micron beam defocus. Spectral images were obtained with the xCLent detection system. Figure 3 shows a high resolution panchromatic CL image demonstrating fine features in the cement overgrowths. Figure 4 shows an image of the cements determined using a selected portion of the pixels, and on a Gaussian fitted spectral map, peak $2.815 \mathrm{eV}$. UW images were acquired on the Hitachi W filament S3400N VP SEM operated at $10 \mathrm{kV}$ using approximately $300 \mathrm{~ms}$ dwell time. Figure 5 shows a BSE image demonstrating the compositional continuity of the quartz. Figure 6 is a CL image clearly delineating cement overgrowths up to $60 \mu \mathrm{m}$ thick in dark blue from the underlying quartz.

[1] S Boggs, D Krinsley in "Application of Cathodoluminescence Imaging to the Study of Sedimentary Rocks", (Cambridge University Press, Cambridge) p.19

[2] J Fournelle, 2012, Towards a quartz sandstone SEM-CL intensity imaging reference material, MAS Standards Topical Conference, Boulder, CO

[3] M Benson, A Wilson, Frac Sand in the United States, USGS, Open-File Report 2015-1107 


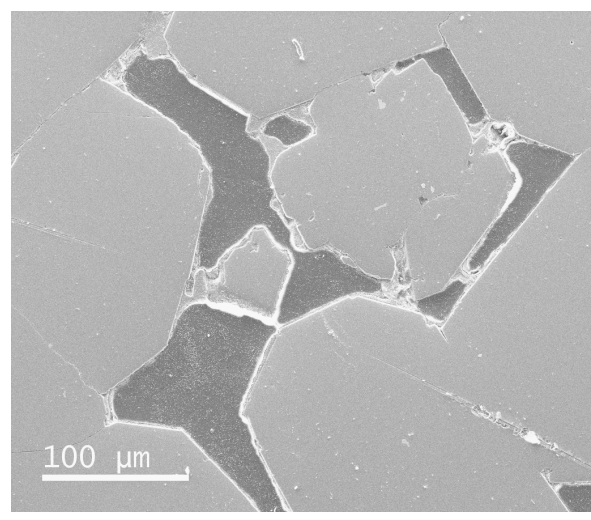

Figure 1. SE Image with FEI Quanta 200 FEG

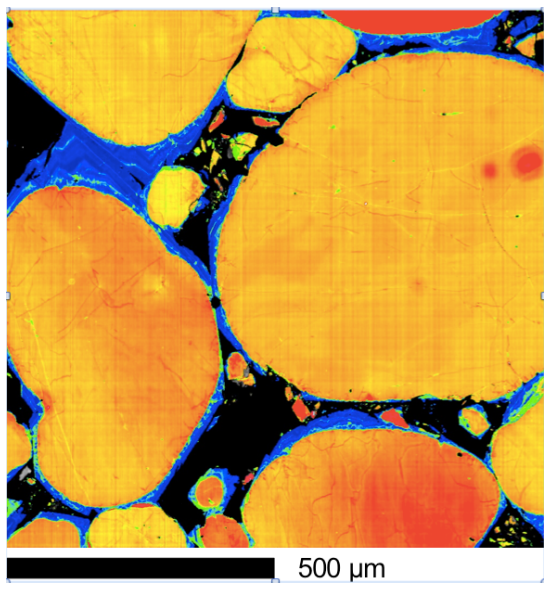

Figure 3. Panchromatic CL with JEOL $8500 \mathrm{~F}$ with $\mathrm{xCLent}$ detector.

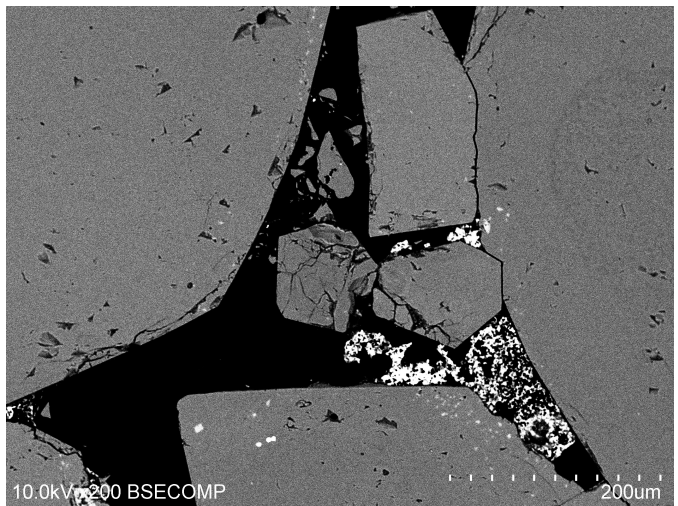

Figure 5. BSE image from UW S3400N Hitachi SEM.

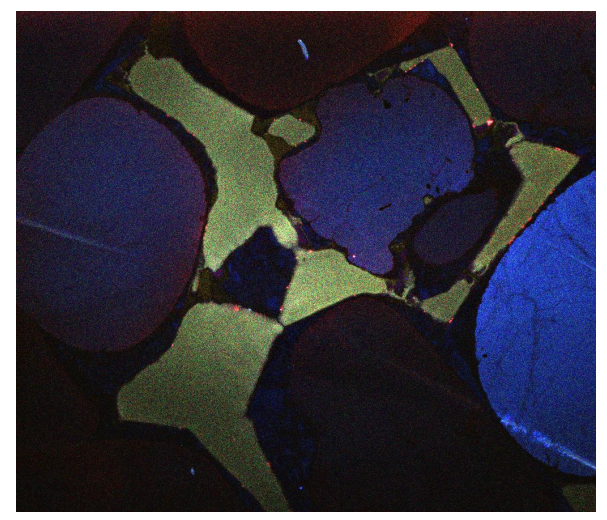

Figure 2. Panchromatic CL with Gatan MonoCL4 Elite with HSPMT.

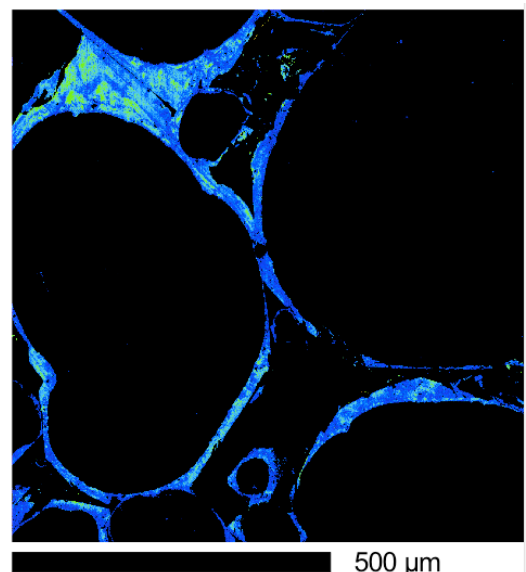

Figure 4. Spectral image at $2.815 \mathrm{eV}$ showing quartz overgrowths.

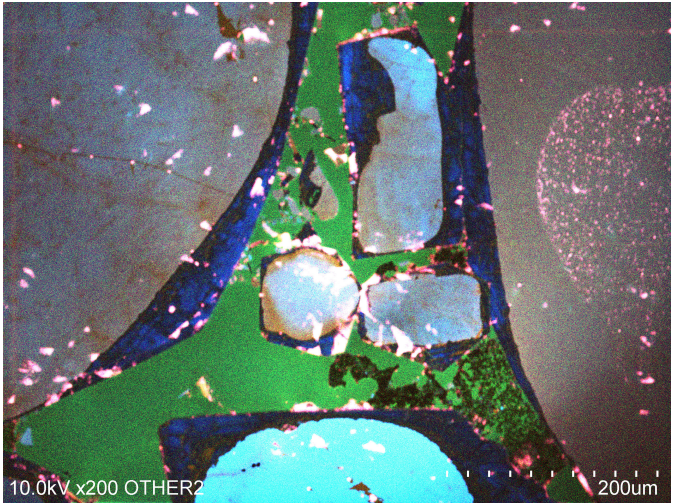

Figure 6. CL image from UW S3400N Hitachi SEM with Gatan PanCL/F. 